\title{
Recovery of Foreign Interest Rates from Exchange Binary Options
}

\author{
M. Mitsuhiro ${ }^{1}$ and Y. Ota ${ }^{2}$ \\ 1. Nikkei Research Inc, 2-2-1 Uchikanda, Chiyoda-ku, Tokyo 101-0047, Japan \\ 2. Department of Culture and Information Science, Doshisha University, 1-3 Tatara Miyakodani Kyotanabe, Kyoto 610-0394, Japan
}

\begin{abstract}
One of most challenging problems from applying the Black-Scholes model to financial derivatives, is reconciling the deviation between the expected and observed values. This study derives an extension of the Black-Scholes model and recovers the real drift of binary call options from their market prices. For space-dependent real drift, we obtain stable linearization and an integral equation. We also find that using market prices of options with different strike prices enables us to identify the term structure of the real drift. Results demonstrate that our new approach can confirm the existence of arbitrage opportunities in a binary option transaction.
\end{abstract}

Key words: Inverse problem, binary option, real drift, arbitrage opportunities, Black-Scholes model.

\section{Introduction}

Financial derivatives are contracts wherein payment is derived from an underlying asset such as a stock, bond, commodity, interest, or exchange rate. An underlying asset $S_{t}$ at time $t$ is modeled by the following stochastic differential equation:

$$
d S_{t}=\mu\left(t, S_{t}\right) S_{t} d t+\sigma\left(t, S_{t}\right) S_{t} d W_{t}(1.1)
$$

where the process $W t$ is the Brownian motion. Parameters $\mu(t, S)$ and $\sigma(t, S)$ are the real drift and local volatility of the underlying asset, respectively. Black and Sholes first found how to construct a dynamic portfolio $\Pi t$ comprising the derivative security and its underlying asset [1]. Their approach is developed in the probability theory, and hedging and pricing theory of the derivative security is established as mathematical finance. By Ito's lemma, the stochastic behavior of the derivative security $u(t, S)$ is governed by the following stochastic differential equation:

$$
\begin{gathered}
d u=\left(\frac{\partial u}{\partial t}+\mu(t, S) S \frac{\partial u}{\partial S}+\frac{1}{2} \sigma(t, S)^{2} \frac{\partial^{2} u}{\partial S^{2}}\right) d t+ \\
\sigma(t, S) S \frac{\partial u}{\partial S} d W
\end{gathered}
$$

Corresponding author: Yasushi Ota, Ph.D., research fields: differential equation, mathematical finance, econometrics model. E-mail: yota@mail.doshisha.ac.jp.
In the absence of arbitrage opportunities, the instantaneous return of this portfolio must equal the interest rate $r$, the return on a riskless asset, such as a bank deposit. Therefore, this equality takes the form of the following partial differential equation:

$$
\begin{gathered}
\frac{\partial u}{\partial t}+\frac{1}{2} \sigma(t, S)^{2} S^{2} \frac{\partial^{2} u}{\partial S^{2}}+ \\
(r-\delta) S \frac{\partial u}{\partial S}-r u=0
\end{gathered}
$$

where $r$ and the divided rate $\delta$ are the known constants.

Their approach provides a useful simple method of pricing with financial derivatives, risk premium, and default probability estimation under the assumption that the risky asset is log-normally distributed. However, theoretical prices of options with different strike prices calculated by the Black-Scholes model differ from real market prices. Specifically, when we apply the Black-Scholes model to default probability estimation, we must be wary of the deviation between the expected and observed values. Merton has formulated a default probability estimation using a model based on Ref. [1] by considering the value of the firm instead of the stock, the firm's debt instead of strike price, and its equity instead of option price [7]. 
However, as shown in deriving the Black-Scholes model [1], under the no arbitrage property, the real drift $\mu$ does not enter Eq. (1.3). Therefore, if we solve equation Eq. (1.3) with a suitable initial condition, we cannot obtain a solution with the form including $\mu$. In contrast, by Ref. [4], not using the model based on Black and Scholes, the default probability is given by

$$
\operatorname{Pr}\left(S_{T}<K \mid S_{t}\right)=N(-d)=1-N(d)^{\prime} 1.4
$$

with

$$
d=\frac{\ln \left(S_{t} / K\right)+\left(\mu-\sigma^{2} / 2\right)(T-t)}{\sigma \sqrt{T-t}}
$$

where $K$ is the firm's debt at maturity $T$ and $N(d)$ is the cumulative probability distribution for a variable that has a standard normal distribution with mean of zero and standard deviation of one. Note that the drift rate $\mu$ enters Eq. (1.4). This difference arises whenever we apply option theory to default probability estimation. Considering this difference, we derive an extended arbitrage model. We also establish a new mathematical approach to reconstruct real drift from the observable data and confirm the existence of an arbitrage opportunity in the financial markets.

This study is divided into six sections. In Section 2, we derive our new model without an assumption of no arbitrage. In Section 3, we formulate the inverse problem of the binary option pricing. The difficulty with the original problem (IPB) suggests studying a standard linearization procedure, which is the approximate algorithm neglecting terms of higher order with respect to a perturbation. In Section 4 we use the linearization and derive the integral equation for solving approximately this problem. Finally, in Section 5 we discretize this integral equation and provide several numerical examples of recovering a drift function. For a given drift $\mu$ we generate data by solving PDE and by using the generated data we recover the drift $\mu$ from the integral equation. The mathematical accuracy of our numerical results is shown in Appendix.

\section{Derivation of the Arbitrage Model}

By Eqs. (1.1) and (1.2) and the hedging theory (see Ref. [1]), the infinitesimal change $\mathrm{d} \Pi \mathrm{t}$ of the portfolio within time interval $d t$ is:

$$
\begin{gathered}
d \Pi_{t}=d u-\frac{\partial u}{\partial S} d S \\
=\left(\frac{\partial u}{\partial t}+\mu S \frac{\partial u}{\partial S}+\frac{1}{2} \sigma^{2} \frac{\partial^{2} u}{\partial S^{2}}\right) d t-\frac{\partial u}{\partial S} \mu S d t
\end{gathered}
$$

Under the assumption of no arbitrage opportunities, $\mathrm{d} \Pi$ t must equal $r$ as follows:

$$
\begin{aligned}
d \Pi_{t} & =r \Pi_{t} d t \\
& =r u d t-r \frac{\partial u}{\partial S} S d t
\end{aligned}
$$

To derive a new model, we use $\mathrm{d} \Pi \mathrm{t}$ as the following form:

$$
d \Pi_{t}=r u d t-\mu \frac{\partial u}{\partial S} S d t
$$

Although Black and Sholes use Eq. (2.1) under the assumption of absence of arbitrage opportunities, we use Eq. (2.2) because we assume the existence of arbitrage opportunities and, especially, consider that opportunities exist to arbitrage the instantaneous return of an underlying asset. Then we obtain the following equation:

$$
\begin{aligned}
& \frac{\partial u}{\partial t}+\frac{1}{2} \sigma(t, S)^{2} S^{2} \frac{\partial^{2} u}{\partial S^{2}}+ \\
& \mu(t, S) S \frac{\partial u}{\partial S}-r u=0 .
\end{aligned}
$$

This model is a form of arbitrage model. Further, in this study, we consider the case of the binary option model, i.e., the final condition at the maturity specified by

$$
u(T, S)=H(S-K)= \begin{cases}1 & S \geq K \\ 0 & S<K\end{cases}
$$

In Ref. [9], we propose our new mathematical approach for inverse problem in financial markets. Using our model, Refs. [11, 12] discuss the parameter identification problem for a parabolic equation.

When $\mu$ is constant, the solution $U$ of Eqs. (2.3) and 
(2.4) takes the following form:

$$
\begin{gathered}
u(t, S)= \\
\frac{1}{\sqrt{\pi}} e^{-r(T-t)} \int_{\frac{-\ln (S / K)-\left(\mu-\sigma^{2} / 2\right)(T-t)}{\sigma \sqrt{(T-t)}}}^{\infty} e^{-\theta^{2}} d \theta
\end{gathered}
$$

Therefore, our Eqs. (2.3) and (2.4) merit application to a default probability estimation.

\section{Formulation for Problem}

In this section, using our model, we propose a new mathematical approach to the reconstruction of a real drift, called implied drift, from the observable data. We define that the inverse problem of option pricing seeks $\mu$ given

$$
u\left(t^{*}, S^{*} ; T, K\right)=u^{*}(K)
$$

Here, $S^{*}$ is the market price of the underlying asset at time $t^{*}$, and $u^{*}$ denotes the market price of options with different $K$ for a given $T$.

To obtain our results, we use a price $u(t, S ; T, K)$ to satisfy the dual Eq. (2.3) with respect to $K$ and $T$ :

$$
\frac{\partial u}{\partial T}-\frac{1}{2} \sigma^{2} K^{2} \frac{\partial^{2} u}{\partial K^{2}}+\mu(K) K \frac{\partial u}{\partial K}+r u=0
$$

Eq. (3.1) was found by Ref. [5] for Black-Scholes model Eq. (1.3):

$\frac{\partial u}{\partial T}-\frac{1}{2} \sigma^{2} K^{2} \frac{\partial^{2} u}{\partial K^{2}}+(r-\delta) K \frac{\partial u}{\partial K}+r u=0$ Where $K$ is the strike price at maturity. The last equation can recover the local volatility from market prices of call options with different maturities and strike prices. In general, the parameter identification for a parabolic equation is ill-posed; however, we know conditions that ensure the local volatility's identification problem is well-posed.

Bouchoev and Isakov formulated how to identify $\sigma(K)$ from the information of $u(t, S ; T, K)$ for all possible strike prices, albeit for a single maturity [2]. A standard linearization procedure for this identification problem is used in Refs. [2, 3]. Now, we consider that a price $u(T, K)$ of a binary option with maturity $T$ and strike price $K$ satisfies

$$
\left\{\begin{array}{cc}
\frac{\partial u}{\partial T}-\frac{1}{2} \sigma^{2} K^{2} \frac{\partial^{2} u}{\partial K^{2}}+\mu(K) K \frac{\partial u}{\partial K}+r u=0, & (T, K) \in(0, t) \times(0, \infty) \\
\left.u(t, S ; T, K)\right|_{T=t}=H(S-K), & K \in(0, \infty),
\end{array}\right.
$$

and make the changes in variables $y=\ln \left(K / S^{*}\right), \tau=$ $T-t$. Then, we arrive at the following problem: parameters $\sigma, r$ and $\tau^{*}=T-t^{*}$. From the option price $U^{*}(y), y \in I$, we identify the $\operatorname{drift} \mu(y)$ satisfying

Inverse Problem of Binary option (IPB). We know

$$
\left\{\begin{array}{cc}
\frac{\partial U}{\partial \tau}-\frac{1}{2} \sigma^{2} \frac{\partial^{2} U}{\partial y^{2}}-\left(\frac{1}{2} \sigma^{2}-\mu(y)\right) \frac{\partial U}{\partial y}+r U=0, & (\tau, y) \in\left(0, \tau^{*}\right) \times \boldsymbol{R} \\
\left.U\left(t, S^{*} ; \tau, y\right)\right|_{\tau=0}=H(-y), & y \in \boldsymbol{R} \\
U\left(\tau^{*}, y\right)=U^{*}(y), & y \in \boldsymbol{I} .
\end{array}\right.
$$

\section{Linearization for Inverse Problem}

First, we assume that

$$
\mu(y)=\mu_{0}+f(y)
$$

Where $\mu_{0}$ is constant and $f(y)$ denotes a small perturbation, and

$$
U=U_{0}+V \text {. }
$$

Here $U 0$ solves the Cauchy problem Eq. (3.2) with $\mu(y)=\mu_{0}$ and $V$ is the term depending on the perturbation $f$. Then, in order to obtain the linearized algorithm, observe the estimation 


$$
\begin{aligned}
0=\frac{\partial U}{\partial \tau}- & \frac{1}{2} \sigma^{2} \frac{\partial^{2} U}{\partial y^{2}}-\left(\frac{1}{2} \sigma^{2}-\mu(y)\right) \frac{\partial U}{\partial y}+r U \\
& \quad-\left\{\frac{\partial U_{0}}{\partial \tau}-\frac{1}{2} \sigma^{2} \frac{\partial^{2} U_{0}}{\partial y^{2}}-\left(\frac{1}{2} \sigma^{2}-\mu_{0}\right) \frac{\partial U_{0}}{\partial y}+r U_{0}\right\} \\
= & \frac{\partial V}{\partial \tau}-\frac{1}{2} \sigma^{2} \frac{\partial^{2} V}{\partial y^{2}}-\left(\frac{1}{2} \sigma^{2}-\mu_{0}\right) \frac{\partial V}{\partial y}+r V+\frac{\partial U_{0}}{\partial y} f(y)+\frac{\partial V}{\partial y} f(y)
\end{aligned}
$$

Neglecting the quadratically small term with $\frac{\partial V}{\partial y} f(y)$ respect to $f, V$ satisfies

$$
\left\{\begin{array}{cc}
\frac{\partial V}{\partial \tau}-\frac{1}{2} \sigma^{2} \frac{\partial^{2} V}{\partial y^{2}}-\left(\frac{1}{2} \sigma^{2}-\mu_{0}\right) \frac{\partial V}{\partial y}+r V=-\frac{\partial U_{0}}{\partial y} f(y), \quad(\tau, y) \in\left(0, \tau^{*}\right) \times \boldsymbol{R} \\
\left.V(\tau, y)\right|_{\tau=0}=0, & y \in \boldsymbol{R} \\
\left.V(\tau, y)\right|_{\tau=\tau^{*}}=U^{*}(y)-U_{0}(y), & y \in \mathbf{I} .
\end{array}\right.
$$

The new substitution

$$
W(\tau, y)=e^{-\alpha \tau-\beta y} V(\tau, y)
$$

simplifies Eq. (4.1) to

where

$$
\left\{\begin{array}{cc}
\frac{\partial W}{\partial \tau}-\frac{1}{2} \sigma^{2} \frac{\partial^{2} W}{\partial y^{2}}=-\frac{\partial U_{0}}{\partial y} f(y) e^{-\alpha \tau-\beta y}, & (\tau, y) \in\left(0, \tau^{*}\right) \times \boldsymbol{R} \\
\left.W(\tau, y)\right|_{\tau=0}=0, & y \in \boldsymbol{R},
\end{array}\right.
$$

$$
\alpha=-\frac{\sigma^{2}}{8}-\frac{\mu^{2}}{2 \sigma^{2}}+\frac{\mu}{2}-r, \quad \beta=\frac{\mu}{\sigma^{2}}-\frac{1}{2}
$$

Therefore, we obtain a new problem provided reconstruction of $\mu(y)$.

Linearized Inverse Problem of Binary option (LIPB). We know parameters $\tau^{*}, \mu$, $\sigma$, and $r$. From the option price $V^{*}(y)=U^{*}(y)-U_{0}\left(\tau^{*}, y\right)$, identify the perturbation $f(y)$ satisfying

$$
\left\{\begin{array}{c}
\frac{\partial V}{\partial \tau}-\frac{1}{2} \sigma^{2} \frac{\partial^{2} V}{\partial y^{2}}-\left(\frac{1}{2} \sigma^{2}-\mu_{0}\right) \frac{\partial V}{\partial y}+r V=-\frac{\partial U_{0}}{\partial y} f(y) \\
\left.V(\tau, y)\right|_{\tau=0}=0 \\
V\left(\tau^{*}, y\right)=V^{*}(y) .
\end{array}\right.
$$

The new substitution

$$
W(\tau, y)=e^{-\alpha \tau-\beta y} V(\tau, y)
$$

Simplifies Eq. (4.4) to 


$$
\left\{\begin{array}{c}
\frac{\partial W}{\partial \tau}-\frac{1}{2} \sigma^{2} \frac{\partial^{2} W}{\partial y^{2}}=-\frac{\partial U_{0}}{\partial y} f(y) e^{-\alpha \tau-\beta y} \\
\left.W(\tau, y)\right|_{\tau=0}=0
\end{array}\right.
$$

where

$$
\alpha=-\frac{\sigma^{2}}{8}-\frac{\mu^{2}}{2 \sigma^{2}}+\frac{\mu}{2}-r, \quad \beta=\frac{\mu}{\sigma^{2}}-\frac{1}{2} .
$$

From Lemma 7.1 in Appendix and the formula for the fundamental solution to the heat equation (see Ref. [6]), we obtain the following result.

Theorem 4.1. Let us denote by $A f$ the solution to Eq. (4.6):

$$
A f=W\left(\tau^{*}, x\right) \text {. }
$$

Then, we have the following equation:

$$
A f(x)=\int_{\mathbb{R}} K\left(x, y ; \tau^{*}\right) f(y) d y
$$

where

$$
K\left(x, y ; \tau^{*}\right)=\frac{1}{2 \sigma^{2}} \operatorname{erfc}\left(\frac{|x-y|+|y|}{\sigma \sqrt{2 \tau}}\right)=\frac{1}{\sigma^{2} \sqrt{\pi}} \int_{\frac{|x-y|+|y|}{\sigma \sqrt{2 \tau^{*}}}}^{\infty} e^{-\theta^{2}} d \theta
$$

Proof. According to the formula for the fundamental solution to the heat Eq. (4.6), $W(\tau, \mathrm{y})$ is described by means of $K(x, y ; s)$ as the following formula:

$$
\begin{gathered}
W(\tau, x)=\int_{\mathbf{R}} K(x, y ; s) f(y) d s d y \\
K(x, y ; \tau)=\int_{0}^{\tau} \frac{1}{\sigma \sqrt{2 \pi(\tau-s)}} e^{-\frac{|x-y|^{2}}{2 \sigma^{2}(\tau-s)}} \frac{1}{\sigma \sqrt{2 \pi s}} e^{-\frac{|y|^{2}}{2 \sigma^{2} s}} d s
\end{gathered}
$$

We simplify $K(x, y ; \tau)$ by using the Laplace transform $G(p)=L(g)(p)$ of $g(\tau)$ with respect to $\tau$. Since the Laplace transform of the convolution is the product of Laplace transforms of convoluted functions, we have

$$
\begin{aligned}
\mathcal{L} K(x, y ; \tau) & =\frac{1}{2 \pi \sigma^{2}} \mathcal{L}\left(\frac{1}{\sqrt{\tau}} e^{-\frac{|x-y|^{2}}{2 \sigma^{2} \tau}}\right) \mathcal{L}\left(\frac{1}{\sqrt{\tau}} e^{-\frac{|y|^{2}}{2 \sigma^{2} \tau}}\right) \\
& =\frac{1}{2 \pi \sigma^{2}} \sqrt{\frac{\pi}{p}} e^{-\frac{\sqrt{2}|x-y|}{\sigma} \sqrt{p}} \sqrt{\frac{\pi}{p}} e^{-\frac{\sqrt{2}|y|}{\sigma} \sqrt{p}} \\
& =\frac{1}{2 \sigma^{2}} \frac{1}{p} e^{-\frac{\sqrt{2}(|x-y|+|y|)}{\sigma} \sqrt{p}}
\end{aligned}
$$

where we use the formula for the Laplace transform of

$$
\mathcal{L}\left(\frac{1}{\sqrt{\tau}} e^{\frac{a^{2}}{4 \tau}}\right)=\frac{1}{\sqrt{p}} e^{-a \sqrt{p}}
$$

Next, applying the formula for the inverse Laplace transform of

$$
\mathcal{L}^{-1}\left(\frac{1}{p} e^{-a \sqrt{p}}\right)=\operatorname{erfc}\left(\frac{a}{2 \sqrt{\tau}}\right)
$$

to the function

$$
\frac{1}{p} e^{-a \sqrt{p}} .
$$


we arrive at

$$
\begin{aligned}
K(x, y ; \tau) & =\mathcal{L}^{-1} \mathcal{L} K(x, y ; \tau) \\
& =\frac{1}{2 \sigma^{2}} \mathcal{L}^{-1}\left(\frac{1}{p} e^{-\frac{\sqrt{2}(|x-y|+|y|)}{\sigma} \sqrt{p}}\right) \\
& =\frac{1}{2 \sigma^{2}} \operatorname{erfc}\left(\frac{|x-y|+|y|}{\sigma \sqrt{2 \tau}}\right) .
\end{aligned}
$$

Thus, the proof is completed.

\section{Numerical Example}

In this section, we propose and numerically test our algorithm for reconstruction of a real drift from several discrete option prices. Moreover, using our algorithm we confirm the existence of arbitrage opportunities.

Here, our algorithm is a method to reconstruct a trend coefficient from observed data using the integral Eq. (4.7) derived in Theorem 4.1. A discrete representation of the integral Eq. (4.7) is given by the method in Ref. [8] as

$$
W\left(\tau^{*}, x_{j}\right) \approx \sum_{i=1}^{n} K\left(x_{j}, y_{i} ; s\right) f\left(y_{i}\right) \Delta_{y}
$$

where $y_{i}(i=1, \ldots, n)$ are points of some interval $I$, $\Delta y=1 / \mathrm{n}$ and the points $x_{j}$ are the measurements points. Here, $K\left(x_{j}, y_{i} ; s\right)$ are the following form:

$$
K\left(x_{j}, y_{i} ; s\right)=\frac{1}{2 \sigma^{2}} \operatorname{erfc}\left(\frac{\left|x_{j}-y_{i}\right|+\left|y_{i}\right|}{\sigma \sqrt{2 \tau^{*}}}\right)
$$

Now, we consider the problem of finding the trend coefficient $f$ satisfying the equation

$$
\boldsymbol{W}=\boldsymbol{K} f
$$

Where

$$
\begin{gathered}
\boldsymbol{W}=\left(W\left(x_{1}, \tau^{*}\right), W\left(x_{2}, \tau^{*}\right),\right. \\
\left.\quad \cdots, W\left(x_{m}, \tau^{*}\right)\right)^{t} \\
\boldsymbol{f}=\left(f\left(y_{1}\right), f\left(y_{2}\right), \cdots f\left(y_{n}\right)\right)^{t}
\end{gathered}
$$

and $\mathrm{K}$ are $m \times n$ matrices of which $(j, i)$ )-entry is expressed by Eq. (5.1).

In this section, we consider the following minimization problem: find $f$ that minimizes the functional

$$
\|\boldsymbol{K} f-W\|^{2}
$$

\subsection{Numerical Algorithm and Its Testing}

To verify the effectiveness of our algorithm, we will solve the minimization problem Eq. (5.5) with the data $W\left(\tau^{*}, x_{j}\right)(j=1, \ldots, m)$ equal to:

$$
e^{-\alpha \tau^{*}-\beta y}\left(U^{*}\left(x_{j}\right)-U_{0}^{*}\left(x_{j}\right)\right)
$$

where $U$ solves the parabolic Eq. (3.2) with $\mu(y)=$ $\mu_{0}+f(y)$ and $U_{0}$ solves the parabolic Eq. (3.2) with $\mu_{0}$ instead of $\mu(y)$.

Now, we will test that we can reconstruct the function $f(y)$ from $W\left(\tau^{*}, x_{j}\right)(j=1, \ldots, m)$ by using our algorithm, particularly, in the case of $f(y)=y$. First, we assume the volatility $\sigma=1$, the interest rate $\mathrm{r}=0$, and $\mu_{0}=0$. Then the direct problem Eq. (3.2) is solved numerically, by the Crank-Nicholson scheme with 81 grid points on some interval $I$, as follows:

$$
\begin{gathered}
a_{j} U_{i+1, j+1}+ \\
\left(1+b_{j}\right) U_{i+1, j}+c_{j} U_{i+1, j-1}= \\
-a_{j} U_{i, j+1}+\left(1-b_{j}\right) U_{i, j}-c_{j} U_{i, j-1}(5.6)
\end{gathered}
$$

where

$$
\begin{gathered}
a_{j}=-\frac{\Delta \tau}{4(\Delta y)^{2}}\left\{1+\Delta y\left(\frac{1}{2}-y_{j}\right)\right\} \\
b_{j}=\frac{\Delta \tau}{2(\Delta y)^{2}},
\end{gathered}
$$

$$
c_{j}=-\frac{\Delta \tau}{4(\Delta y)^{2}}\left\{1-\Delta y\left(\frac{1}{2}-y_{j}\right)\right\}
$$

$y_{i}(i=1,2, \ldots, 81)$ are points of the interval $(-1,1)$ with artificial boundary conditions $U_{i, 0}=1$ and $U_{i, N}=$ 0 . Thereafter, the discrete representation of the direct problem Eq. (3.2) can be written:

$$
\left\{\begin{array}{c}
\boldsymbol{u}_{i+1}=\boldsymbol{A}^{-1} \boldsymbol{B} \boldsymbol{u}_{i}-2 c_{1} \boldsymbol{A}^{-1} \boldsymbol{e}_{1} \\
\boldsymbol{u}_{i}=H(-y),
\end{array}\right.
$$

where $u_{i}=\left(U_{1,1}, U_{1,2}, \ldots, U_{1, N-1}\right)^{\mathrm{T}}, e_{1}=(1,0, \ldots$, $0)^{\mathrm{T}}$ and 


$$
\begin{gathered}
\boldsymbol{A}=\left(\begin{array}{cccccc}
1+b_{1} & a_{1} & 0 & 0 & \cdots & 0 \\
c_{2} & 1+b_{2} & a_{2} & 0 & \cdots & 0 \\
0 & c_{3} & 1+b_{3} & a_{3} & \cdots & 0 \\
\vdots & & \ddots & \ddots & \ddots & \vdots \\
0 & & & c_{N-2} & 1+b_{N-2} & a_{N-2} \\
0 & \cdots & & 0 & c_{N-1} & 1+b_{N-1}
\end{array}\right), \\
\boldsymbol{B}
\end{gathered}
$$

Next we consider solving $U 0$ of the parabolic Eq. (3.2) with $\mu_{0}$ instead of $\mu(y)$.

Substituting

$$
W_{0}(\tau, y)=e^{-\alpha \tau-\beta y} U_{0}(\tau, y)
$$

transforms Eq. (3.2) with $\mu 0$ instead of $\mu(\mathrm{y})$ into

$$
\left\{\begin{array}{c}
\frac{\partial W_{0}}{\partial \tau}-\frac{1}{2} \sigma^{2} \frac{\partial^{2} W_{0}}{\partial y^{2}}=0 \\
\left.W(\tau, y)\right|_{\tau=0}=e^{-\beta y} H(-y)
\end{array}\right.
$$

Then, by the formula for the fundamental solution to the heat Eq. (5.8), we can obtain immediately

$$
\begin{gathered}
U_{0}\left(\tau^{*}, y\right)= \\
\frac{1}{\sqrt{\pi}} e^{-r \tau} \operatorname{erfc}\left(\frac{y-\left(\mu_{0}-\frac{1}{2} \sigma^{2}\right) \tau^{*}}{\sigma \sqrt{2 \tau^{*}}}\right)
\end{gathered}
$$

Using the solution of Eq. (5.7) (i.e., the numerical solution of the direct problem in Eq. (3.2)) and Eq. (5.9), we can solve problem in Eq. (5.5). Consequently, we set $N=81, \Delta \mathrm{y}=0.025$, and $\Delta \tau=$ 0.001 .

Fig. 1 shows the numerical solution $f(y)$ over $y$ using MATLAB. From Fig. 1, we see that the reconstruction is almost perfect, near the center (i.e., at-the-money).

\subsection{Example with Real Data}

Next, we identify the real drift from the market data, which are the binary option prices of foreign exchange between the Euro and Japanese-Yen at 8 p.m. on April 19,2012 . At this time, $S^{*}=106.97$, and maturity is at midnight on April 20, 2012. Market prices of the binary call option with strike prices $K=105.1 \sim 108.3$ are given in Table 1.

Fig. 2 shows the numerical solution $\mu(K)$ over strike prices $K$ using MATLAB.

From this numerical example, we observe that $\mu(K)$ falls to the right from the near point of $K=106.97$, which is the current value of foreign exchange at 8 p.m. on April 19, 2012. Thus, Fig. 2 indicates two facts. First, $\mu$ is not equal interest rate $\mathrm{r}$ (i.e., $\mu$ is not a constant). Second, there is an arbitrage opportunity in this market. The existence of the arbitrage opportunity is confirmed by our new approach to an inverse problem in financial markets.

\section{Conclusion}

This paper has formulated a new approach to an inverse problem in financial markets. 


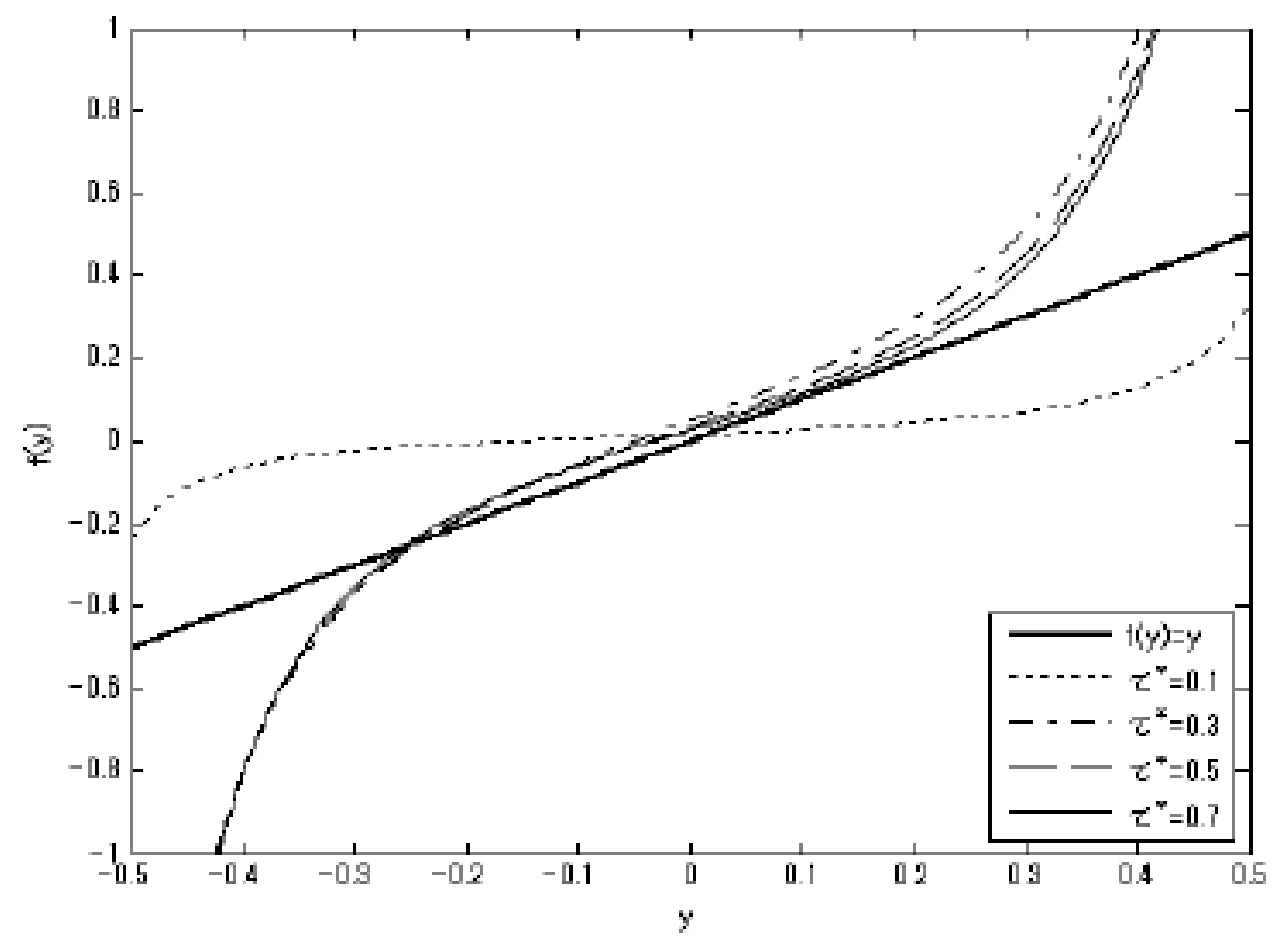

Fig. 1 Recovered linearly perturbation.

Table 1 Real data.

\begin{tabular}{|c|c|c|c|c|c|c|c|c|c|c|c|c|c|c|c|c|c|}
\hline $\bar{K}$ & 105.1 & 105.3 & 105.5 & 105.7 & 105.9 & 106.1 & 106.3 & 106.5 & 106.7 & 106.9 & 107.1 & 107.3 & 107.5 & 107.7 & 107.9 & 108.1 & 108.3 \\
\hline Price & 100.0 & 100.0 & 100.0 & 100.0 & 100.0 & 97.9 & 93.6 & 86.4 & 75.5 & 61.4 & 44.3 & 28.7 & 17.4 & 10.1 & 5.9 & 4.0 & 4.0 \\
\hline
\end{tabular}

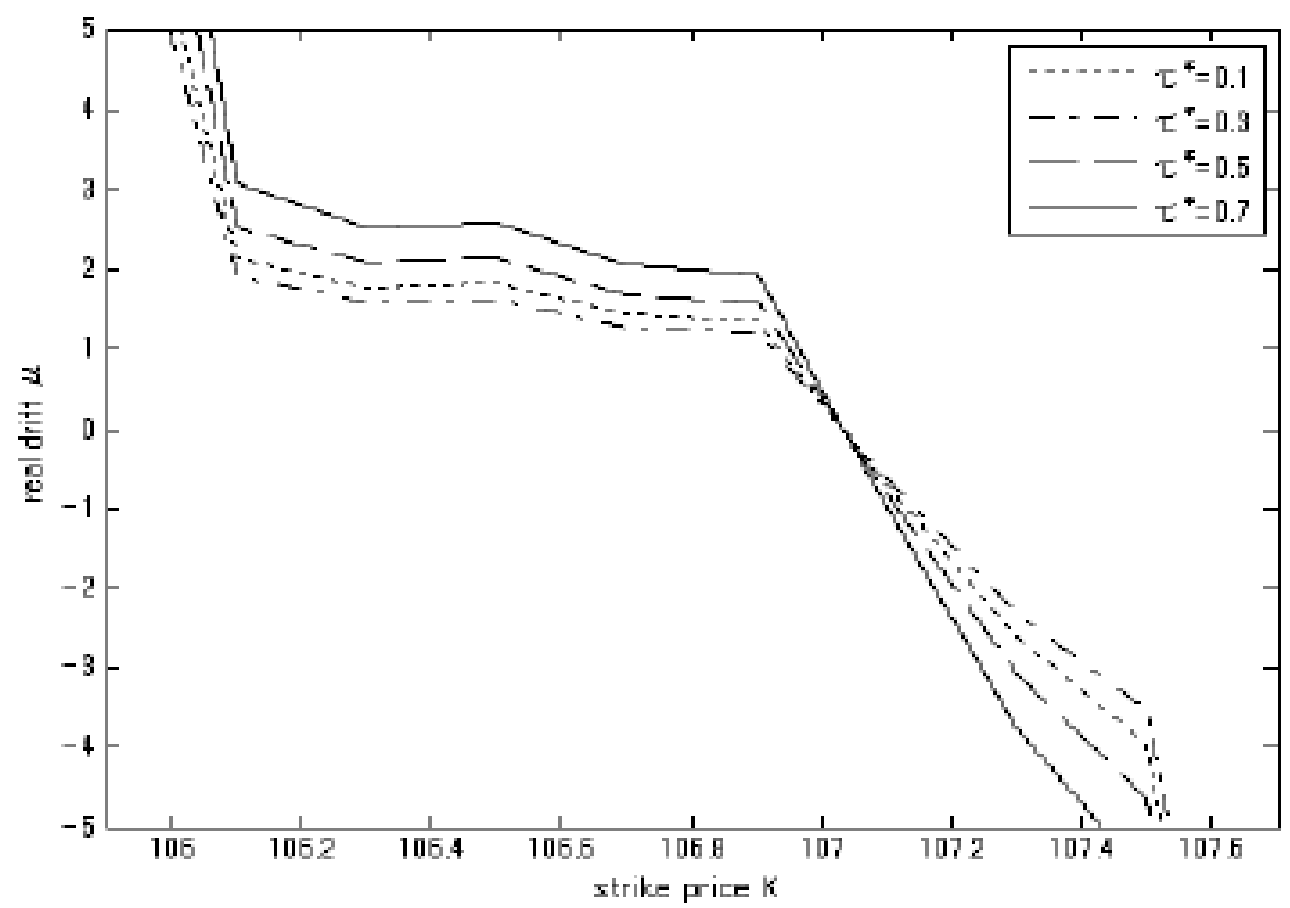

Fig. 2 Identified real drifts. 
Using this approach, we recovered the real drift from the market data and confirmed that an arbitrage opportunity exists in the financial market. However, several problems remain.

For example, the numerical method in Section 5 for solving an inverse problem is in effective. In general, volatility identification in the Black-Scholes model has been observed to be an ill-posed problem. That is, reconstruction of local volatility is unstable because of errors in the data. Similarly, because the identification of real drift in our model is also an ill-posed problem, we need a way to stabilize the inverse problem when applying a numerical method. Tikhonov regularization is one of several methods for doing so. Our next step is to apply it to our inverse problem.

To conclude, although we consider only binary options, we hope to adjust our new approach to include real drift of American options and basket options with two variables by changing boundary conditions. Thus, it is important to study these practical cases.

\section{References}

[1] Black, F., and Sholes, M. 1973. "The Pricing of Options and Corporate Liabilities." Journal of Political Economy 81: 637-59.

[2] Bouchouev, I., and Isakov, V. 1999. "Uniqueness, Stability and Numerical Methods for the Immerse Problem That Arises in Financial Markets. Inverse
Problems 15: R95-R116.

[3] Bouchouev, I., Isakov, V., and Valdivia, N. 2002. "Recovery of Volatility Coefficient by Linearization." Quantitive Finance 2: 257-63.

[4] Bomies, A. J. 1964. "Elements of a Theory of Stock-Option Value" J. Political Econ. 72 (2): 163-75.

[5] Dupire, B. 1994. "Pricing with a Smile." Risk 7: 18-20.

[6] Friedman, A. 1964. Partial Differential Equations of Parabolic Type. Prentice-Hall Englewood Clifts N.J.

[7] Robert, M. 1974. "On the Pricing of Corporate Debt: The Risk Stricture of Interest Rates" Journal of Finance 29: 449-70.

[8] Imai, H., and Takeuchi, T. 2001. "Some Advanced Applications of the Spectral Collocation Method." GAKUTO Int.. Ser. Math. Appl. 17: 323-35.

[9] Mitsuhiro, M., Ota, Y., and Yadohisa, H. 2012. "New Mathematical Approach for an Inverse Problem in Financial Markets.” Proceedings of COMPSTAT 2012, 585-94.

[10] Ota, Y., and Kaji, S. 2014. Reconstruction of Local Volatility for the Binary Option Model J. Inverse Ill-posed probl. (to apper).

[11] Korolev, M., Kubo, H., and Yagola, G. 2012. Parameter Identification Problem for a Parabolic Equation-Application to the Black-Scholes Option Pricing Model J. Inverse Ill-posed probl. 20 No. 3 327-37.

[12] Doi, S., and Ota, Y. 2014. An Application of Microlocal Analysis to an Immerse Problem Arising from Financial Markets. arXiv:1404.7018vl (preprint).

[13] Jari, K., and Erkki, S. 2014. "Statistical and Computational Immerse Problems." Applied Mathematical Science 160. Springer.

[14] Wand, Y., Yagola, G., and Yang, C. 2010. Optimization and Regularzation for Computational Immerse Problems and Applications. Springer. 


\section{Appendix}

In this section, we prove the uniqueness of the solution of linearized inverse problem of binary options (LIPB). Theorem 6.1 is important because we can guarantee that only one solution exists if there is a numerical solution for LIPB. However, the method of the proof is slightly technical.

First, to compute the function

$$
\frac{\partial U_{0}}{\partial y}(\tau, y)
$$

we prove the following lemma.

Lemma A1. $U_{0}$ solves Eq. (3.2) with $\mu(y)=\mu_{0}$. Then,

admits the following form

$$
\frac{\partial U_{0}}{\partial y}(\tau, y)
$$

$$
\frac{\partial U_{0}}{\partial y}=-\frac{1}{\sigma \sqrt{2 \pi \tau}} e^{-\frac{(y-x)^{2}}{2 \sigma^{2} \tau}+\alpha \tau+\beta y} .
$$

Proof. We begin with the substitution

$$
\frac{\partial U_{0}}{\partial y}=W_{0} e^{\alpha \tau+\beta y},
$$

which Eq. (3.2) with $\mu(y)=\mu_{0}$ into

$$
\left\{\begin{array}{c}
\frac{\partial W_{0}}{\partial \tau}-\frac{1}{2} \sigma^{2} \frac{\partial^{2} W_{0}}{\partial y^{2}}=0, \\
\left.W_{0}(\tau, y)\right|_{\tau=0}=-e^{-\beta y} \delta(y),
\end{array}\right.
$$

we have

$$
\begin{aligned}
W_{0}(\tau, y) & =\int_{\mathbb{R}}-e^{-\beta x} \delta(x) \frac{1}{\sqrt{2 \pi \sigma^{2} \tau}} e^{-\frac{(y-x)^{2}}{2 \sigma^{2} \tau}} d x \\
& =-\frac{1}{\sqrt{2 \pi \sigma^{2} \tau}} \int_{\mathbb{R}} \delta(x) e^{-\beta x} e^{-\frac{(y-x)^{2}}{2 \sigma^{2} \tau}} d x \\
& =-\frac{1}{\sqrt{2 \pi \sigma^{2} \tau}} e^{-\frac{y^{2}}{2 \sigma^{2} \tau}} \\
\frac{\partial U_{0}}{\partial y} & =-\frac{1}{\sigma \sqrt{2 \pi \tau}} e^{-\frac{y^{2}}{2 \sigma^{2} \tau}+\alpha \tau+\beta y} .
\end{aligned}
$$

Thus, the proof is completed.

Finally, we prove the uniqueness of the solution of LIPB.

Theorem A1.

Let $I=(-\alpha, \alpha)$ and $\omega_{0}$ be the root of the equation

$$
2 \omega-e^{-2 \omega}-3=0
$$

If

$$
\frac{2 \alpha^{2}}{\tau^{*} \sigma^{2}}<\omega_{0}
$$


then the solution $f \in L^{\infty}(I)$ to LIPB (4.4) is unique.

Proof. To prove the claim of this theorem, it suffices to show the uniqueness of the solution $f$ to the integral Eq. (4.7), i.e., to prove $f=0$ assuming the left-hand side of Eq. (4.7) is 0 .

Differentiating Eq. (4.7) on $I$, we have

$$
\begin{aligned}
\frac{\partial}{\partial x} W\left(\tau^{*}, x\right) & =\frac{\partial}{\partial x} \int_{I} B\left(x, y ; \tau^{*}\right) f(y) d y \\
& =-\frac{1}{\sigma^{3} \sqrt{2 \pi \tau^{*}}} \int_{\mathbf{I}} \operatorname{sign}(x-y) e^{-\frac{(|x-y|+|y|)^{2}}{2 \sigma^{2} \tau^{*}}} d y
\end{aligned}
$$

where we used the following formula

$$
\frac{\partial}{\partial x}|x-y|=\operatorname{sign}(x-y)
$$

Moreover differentiating Eq. (A3)

$$
\begin{aligned}
\frac{\partial^{2}}{\partial^{2} x} W\left(\tau^{*}, x\right)= & -\frac{1}{\sigma^{3} \sqrt{2 \pi \tau^{*}}} \int_{I} \frac{\partial}{\partial x}\left\{\operatorname{sign}(x-y) e^{-\frac{(|x-y|+|y|)^{2}}{2 \sigma^{2} \tau^{*}}}\right\} d y \\
= & -\frac{1}{\sigma^{3} \sqrt{2 \pi \tau^{*}}} \int_{I}\left\{-\frac{1}{\sigma^{2} \tau^{*}}(\operatorname{sign}(x-y))^{2}(|x-y|+|y|)\right. \\
= & -2 \delta(x-y)\} e^{-\frac{(|x-y|+|y|)^{2}}{2 \sigma^{2} \tau^{*}}} f(y) d y \\
= & +\frac{1}{\sigma^{5} \tau^{*} \sqrt{2 \pi \tau^{*}}} \int_{I}(|x-y|+|y|) e^{-\frac{(|x-y|+|y|)^{2}}{2 \sigma^{2} \tau^{*}}} f(y) d y \\
& +\frac{\sqrt{2}}{\sigma^{3} \sqrt{\pi \tau^{*}}} e^{-\frac{|x|^{2}}{2 \sigma^{2} \tau^{*}}} f(x),
\end{aligned}
$$

where we used that $f(y)=0$ outside $I$ and the following formula:

$$
\frac{\partial}{\partial x} \operatorname{sign}(x-y)=\delta(x-y)
$$

Finally, multiplying both the sides by

$$
\frac{\sigma^{3} \sqrt{\pi \tau^{*}}}{\sqrt{2}} e^{\frac{|x|^{2}}{2 \sigma^{2} \tau^{*}}}
$$

we have

$$
\begin{aligned}
f(x)+\frac{1}{2 \sigma^{5} \tau^{*}} \int_{I}(|x-y|+|y|) e^{-\frac{(|x-y|+|y|)^{2}}{2 \sigma^{2} \tau^{*}}} f(y) d y \\
=\frac{\sigma^{3} \sqrt{\pi \tau^{*}}}{\sqrt{2}} e^{\frac{|x|^{2}}{2 \sigma^{2} \tau^{*}}} \frac{\partial^{2}}{\partial^{2} x} W\left(\tau^{*}, x\right)
\end{aligned}
$$

To prove the assertion of the theorem, it suffices to show the uniqueness of the solution to Eq. (A6), i.e., to prove $f=0$ assuming that $W\left(\tau^{*}, x\right)=0$ in the right-hand side of Eq. (A6).

Now, we assume that $W\left(\tau^{*}, x\right)$ in Eq. (A6) is 0 . Thus, 


$$
f(x)+\frac{1}{2 \sigma^{2} \tau^{*}} \int_{I}(|x-y|+|y|) e^{-\frac{(|x-y|+|y|)^{2}}{2 \sigma^{2} \tau^{*}}} f(y) d y=0
$$

and $f \neq 0$. Then,

$$
\|f\|_{\infty}
$$

satisfies the following inequality

$$
\|f\|_{\infty} \leq \frac{1}{2 \sigma^{2} \tau^{*}} \int_{I}\left(\left|x_{0}-y\right|+|y|\right) e^{-\frac{\left(\left|x_{0}-y\right|+|y|\right)^{2}}{2 \sigma^{2} \tau^{*}}}\|f\|_{\infty} d y
$$

where we recall that,

$$
\|f\|_{\infty}(I)
$$

is the essential supremum of $|f|$ over $I$ and assume that

$$
\|f\|_{\infty}(I)=f\left(x_{0}\right)>0
$$

at some $x_{0}$ in $I$. Here, we observe that,

$$
\begin{aligned}
& \int_{I}(|x-y|+|y|) e^{-\frac{(|x-y|+|y|)^{2}}{2 \sigma^{2} \tau^{*}}} d y \\
& =\int_{-\alpha}^{x}(|x-y|+|y|) e^{-\frac{(|x-y|+|y|)^{2}}{2 \sigma^{2} \tau^{*}}} d y+\int_{x}^{0}(|x-y|+|y|) e^{-\frac{(|x-y|+|y|)^{2}}{2 \sigma^{2} \tau^{*}}} d y \\
& +\int_{0}^{x}(|x-y|+|y|) e^{-\frac{(|x-y|+|y|)^{2}}{2 \sigma^{2} \tau^{*}}} d y+\int_{x}^{\alpha}(|x-y|+|y|) e^{-\frac{(|x-y|+|y|)^{2}}{2 \sigma^{2} \tau^{*}}} d y \\
& =\int_{-\alpha}^{x}(x-2 y) e^{-\frac{(x-2 y)^{2}}{2 \sigma^{2} \tau^{*}}} d y+\int_{x}^{0}(-x) e^{-\frac{(-x)^{2}}{2 \sigma^{2} \tau^{*}}} d y \\
& +\int_{0}^{x}(x) e^{-\frac{(x)^{2}}{2 \sigma^{2} \tau^{*}}} d y+\int_{x}^{\alpha}(2 y-x) e^{-\frac{(2 y-x)^{2}}{2 \sigma^{2} \tau^{*}}} d y \\
& =\left(2 x^{2}+\sigma^{2} \tau^{*}\right) e^{-\frac{x^{2}}{2 \sigma^{2} \tau^{*}}}-\frac{\sigma^{2} \tau^{*}}{2}\left(e^{-\frac{(2 \alpha-x)^{2}}{2 \sigma^{2} \tau^{*}}}+e^{-\frac{(x+2 \alpha)^{2}}{2 \sigma^{2} \tau^{*}}}\right) .
\end{aligned}
$$

Therefore, the right-hand side of Eq. (A8) is equal to

$$
\|f\|_{\infty}\left\{\frac{\tau^{*} \sigma^{2}+2 x_{0}^{2}}{2 \tau^{*} \sigma^{2}}-\frac{1}{4}\left(e^{-\frac{\left(2 \alpha-x_{0}\right)^{2}-x_{0}^{2}}{2 \sigma^{2} \tau^{*}}}+e^{-\frac{\left(x_{0}+2 \alpha\right)^{2}-x_{0}^{2}}{2 \sigma^{2} \tau^{*}}}\right)\right\} .
$$

Here we show that

$$
p(x)=\frac{2 x^{2}}{\tau^{*} \sigma^{2}}-\frac{1}{2}\left(e^{-\frac{2 \alpha(x-\alpha)}{\sigma^{2} \tau^{*}}}+e^{-\frac{2 \alpha(x+\alpha)}{\sigma^{2} \tau^{*}}}\right)<1 \quad \text { in } I
$$

Then we have

$$
\|f\|_{\infty}<\|f\|_{\infty}
$$

This is a contradiction. Therefore, we obtain

$$
\|f\|_{\infty}(I)=0 \text {. }
$$

To prove Eq. (A10), we show that the maximum of $p$ on $\overline{\mathrm{I}}$ is at $x=\alpha$. Then $p(\alpha)$ is the following form:

$$
p(\alpha)=\frac{2 \alpha^{2}}{\tau^{*} \sigma^{2}}-\frac{1}{2}\left(1+e^{-\frac{4 \alpha^{2}}{\tau^{*} \sigma^{2}}}\right) .
$$


Since $\omega_{0}$ is the root of Eq. (A1),

$$
2 \omega_{0}-e^{-2 \omega}=3
$$

and $2 \omega-\mathrm{e}^{-2 \omega}$ is an increasing function, we have

$$
2 \omega-e^{-2 \omega}<2 \omega_{0}-e^{-2 \omega_{0}}=3
$$

Therefore the following inequality

$$
\frac{2 \alpha^{2}}{\tau^{*} \sigma^{2}}-\frac{1}{2}\left(1+e^{-\frac{4 \alpha^{2}}{\tau^{*} \sigma^{2}}}\right)<1
$$

holds by Eq. (A2).

To complete the proof it suffices to prove that

$$
\sup _{x \in I} p(x)=p(\alpha),
$$

but we will reach it by a careful analysis of $p, p^{\prime}, p^{\prime \prime}$ and $p^{\prime \prime \prime}$.

The proof is complete. 